\title{
BRAIN TUMOURS: INCIDENCE, SURVIVAL, AND AETIOLOGY

$\mathrm{T}$ he term "brain tumours" refers to a mixed group of neoplasms originating from intracranial tissues and the meninges with degrees of malignancy ranging from benign to aggressive.

Each type of tumour has its own biology, treatment, and prognosis and each is likely to be caused by different risk factors. Even "benign" tumours can be lethal due to their site in the brain, their ability to infiltrate locally, and their propensity to transform to malignancy. This makes the classification of brain tumours a difficult science and creates problems in describing the epidemiology of these conditions. Public perception generally fails to distinguish between different tumour subtypes and although treatments and prognosis may vary, the functional neurological consequences are frequently similar. This article will give an overview of the burden of brain tumours in the population, looking at the major subtypes where possible, in addition to giving a summary of current views on possible causes.

\section{INCIDENCE}

The descriptions in this paper focus on primary tumours of the brain. It excludes data on spinal cord tumours, metastatic tumours, whose origins are external to the central nervous system, and primary brain lymphomas, which are essentially haematological malignancies.

Malignant tumours of the brain are a rare occurrence accounting for approximately $2 \%$ of all cancers in adults. Approximately 4400 people are newly diagnosed with a brain tumour each year in the UK compared to over 40000 women with breast cancer and approximately 25000 men with prostate cancer. Figure 1 shows additional comparative data for different cancers. The overall annual incidence rate of all brain tumours is 7 per 100000 population. UK data are listed in table 1 .

The greatest proportion of adult tumours are supratentorial, arising in the frontal, temporal and parietal lobes, and the majority (86\%) are gliomas which include astrocytomas, glioblastomas, oligodendroblastomas, and unspecified gliomas.

Threefold differences in the incidence of brain tumours have been reported between countries worldwide and differences are also seen between ethnic groups within the same country. Developed countries appear to have the highest rates of brain tumours but this may be a result of better registration systems which include benign tumours. However, the magnitude of the variation is less compared to other cancers-for example, up to 10-fold differences are seen for breast cancer. Geographical variation has to be cautiously interpreted as, unlike other cancers, the criteria and registration of brain tumours is not always consistent.

The incidence of brain tumours rises with age from approximately 30 years old onwards, in common with virtually all other adult cancers. Figure 2 illustrates the age incidence curve showing a drop in incidence in those over 75 years. It is thought this may well be artefactual and occurring as a consequence of tumours of the brain being less likely to be investigated and detected in the elderly. Symptoms in older people may be explained by other co-morbid conditions such as strokes or physicians may be reluctant to undertake thorough investigations.

Males are more likely to be diagnosed with brain tumours than females, with a male:female ratio of 1.5:1. However, this disguises the fact that women are more likely to develop meningiomas than men.

Despite its relative rarity the burden of these tumours is considerable for the individuals, their families, and the health care system. Poor survival for many tumour types results in a disproportionate number of years of life lost compared to other cancers.

Correspondence to:

Dr Patricia A McKinney, Paediatric Epidemiology Group, Unit of Epidemiology and Health Services Research, University of Leeds, 32 Hyde Terrace, Leeds LS2 9LN, UK p.a.mckinney@leeds.ac.uk

\section{TIME TRENDS}

There have been reports of increasing incidence of primary brain tumours in recent decades which need to be interpreted with caution. Trends over time can only be considered valid when based upon data collected according to the same definitions and reporting practice. Inconsistencies and changes over time may be the explanation for the observed rises which have been attributed to various factors. Improved diagnostic imaging, following introduction of radio isotope imaging, 
Table 1 Brain and central nervous system tumours: UK incidence 1999

\begin{tabular}{|c|c|c|c|c|c|c|}
\hline & & England & Wales & Scotland & Northern Ireland & UK \\
\hline \multirow[t]{3}{*}{ Numbers } & Males & 2092 & 159 & 196 & 81 & 2528 \\
\hline & Females & 1529 & 136 & 165 & 37 & 1867 \\
\hline & Persons & 3.621 & 295 & 361 & 118 & 4395 \\
\hline \multirow[t]{3}{*}{ Age standardised rates* } & Males & 8.3 & 10.1 & 7.6 & 10.4 & 8.4 \\
\hline & Females & 5.3 & 7.6 & 5.2 & 4.4 & 5.4 \\
\hline & Persons & 6.8 & 8.8 & 6.3 & 7.2 & 6.8 \\
\hline \multirow[t]{3}{*}{$95 \%$ confidence intervals } & Males & (8.0 to 8.7$)$ & (8.5 to 11.7 ) & $(6.5$ to 8.6$)$ & (8.1 to 12.7 ) & (8.1 to 8.7$)$ \\
\hline & Females & (5.1 to 5.6$)$ & $(6.4$ to 8.9$)$ & $(4.4$ to 6.0$)$ & $(3.0$ to 5.8$)$ & (5.2 to 5.7$)$ \\
\hline & Persons & $(6.5$ to 7.0$)$ & (7.8 to 9.8$)$ & $(5.6$ to 6.9$)$ & (5.9 to 8.5$)$ & (6.6 to 7.0$)$ \\
\hline
\end{tabular}

*Directly age standardised (European) rate per 100000 population at risk.

Source: www.cancerresearchUK.org

computed tomography, and magnetic resonance imaging in the 1970s and 1980s, will have resulted in higher detection rates and better differential diagnosis of brain tumours which might have previously been diagnosed as strokes or metastatic tumours. Access to services will have improved, making it more likely that a patient with a tumour is registered. In addition, histopathological technology has increased the specificity of tumour diagnosis and thus an apparent increase in specific tumour types-for example, astrocytomas-may merely be a consequence of fewer non-specific diagnoses being registered. In Norway and the USA specific studies investigating whether time trends can be accounted by these factors suggest that the reported increase in brain tumours is likely to be an artefact of changing diagnostic and reporting practice. The levelling off of incidence in the 1990s in the USA supports this assertion. Steady rises in the rates of brain tumours in children under 14 years and the elderly over 70 years are most clearly documented. For children this may be explained by changes in the environment, but the increase over time in older patients is more likely attributed to changes in the delivery of care associated with a greater likelihood of full evaluation and intervention.

\section{SURVIVAL}

The length of survival following diagnosis of a brain tumour is dependent on both the age of the patient, histologic subtype and grade of the tumour, and presenting symptoms. Survival chances have improved gradually over the last 30 years but remain poor; for all adults diagnosed with a malignant brain tumour in England and Wales during 1986$90,30 \%$ survived to one year and $15 \%$ to five years. For patients diagnosed between $1981-85$ only $8 \%$ survived to 10 years.

There are known to be geographical differences in the delivery of care to cancer patients within the UK and this may be reflected in long term survival. Recent comparisons of survival from adult brain tumours in eight former National Health Service (NHS) regions (in 1999) and Wales have shown differences between regions and over time within a region. Differences may be accounted for by temporal changes in registration practice, but one particular aspect which is difficult to explain is the observed variation in survival for men and women within a region. It seems unlikely that care and treatment would vary according to sex for patients living in the same area and these differences

\begin{tabular}{|c|c|c|c|}
\hline & Breast & 41300 & $(15 \%)$ \\
\hline & Lung & 38190 & $(14 \%)$ \\
\hline & Large bowel & 35410 & $(13 \%)$ \\
\hline Prostate & & 24710 & $(9 \%)$ \\
\hline Bladder & & 12470 & $(5 \%)$ \\
\hline Stomach & & 9750 & $(4 \%)$ \\
\hline Non-Hodgkin's lymphoma & & 9010 & $(3 \%)$ \\
\hline Head and neck & & 7780 & $(3 \%)$ \\
\hline Oesophagus & & 7230 & $(3 \%)$ \\
\hline Pancreas & & 6990 & $(3 \%)$ \\
\hline Ovary & & 6790 & $(3 \%)$ \\
\hline Leukaemia & & 6660 & $(2 \%)$ \\
\hline Kidney & & 6000 & $(2 \%)$ \\
\hline Malignant melanoma & & 5990 & $(2 \%)$ \\
\hline Body of uterus & & 5200 & $(2 \%)$ \\
\hline Brain and $\mathrm{CNS}$ & & 4400 & $(2 \%)$ \\
\hline Multiple myeloma & & 3350 & $(1 \%)$ \\
\hline$\Longrightarrow$ Cervix & & 3200 & $(1 \%)$ \\
\hline Other & & 32850 & $(13 \%)$ \\
\hline Persons: all malignant neoplasms excluding nor & ASC) & 267450 & $(100 \%)$ \\
\hline
\end{tabular}

Figure 1 UK incidence 1999: cancers which contribute $1 \%$ or more to total cancer burden. 


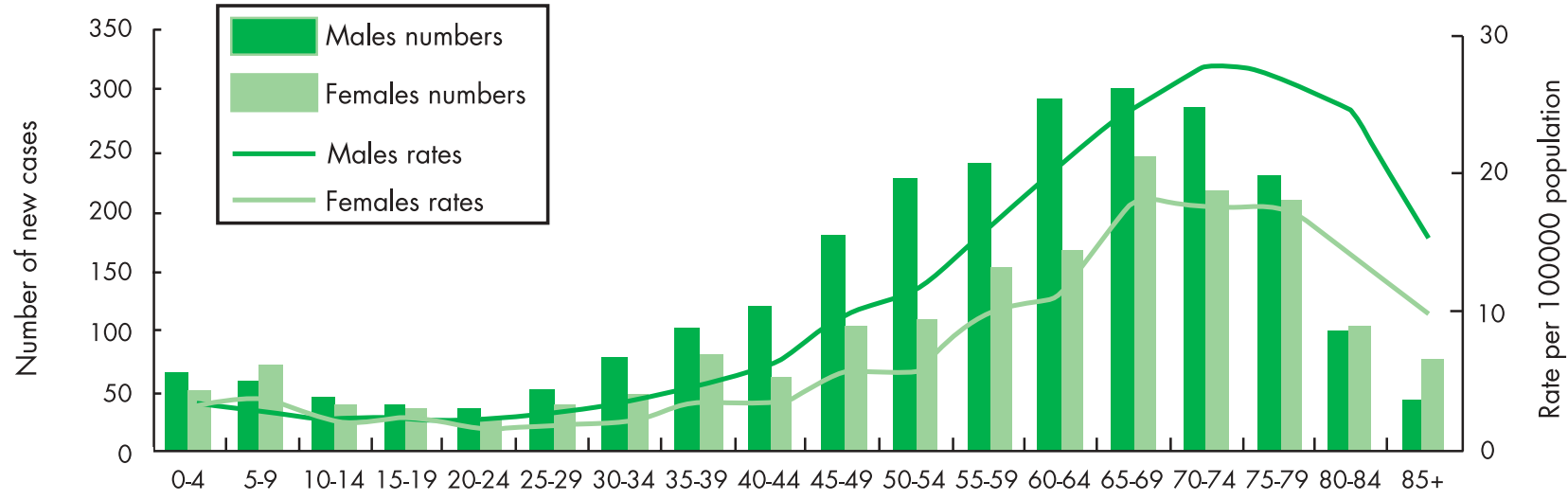

Age at diagnosis

Figure 2 Number of new cases diagnosed and age specific rates per 100000 population, brain and central nervous system cancer, by sex, UK, 1999. Source: www.cancerresearchUK.org.

remain unexplained. Levels of social class measured in deprivation categories are seen to influence survival, with those living in more affluent areas generally having improved survival with the effect seen most prominently at one year post-diagnosis.

Geographical differences in survival exist between countries. The five year survival of patients diagnosed with any type of brain tumour in England, Wales, and Scotland is approximately $13 \%$ in men and $16 \%$ in women. Across Europe equivalent figures are $17 \%$ and $20 \%$ in men and women, respectively, and generally the UK figures are $10 \%$ below the USA. The discrepancy is potentially explained by the fact that figures from the USA include tumours classified as benign which would not be registered in many of the UK cancer registration schemes.

The overall poor survival for this group of tumours masks differences for subtypes. Meningiomas, both benign and malignant, for example, have a much better prognosis whereas glioblastoma multiforme (GBM) has the poorest survival in all age groups.

Other factors associated with survival chances include age, with younger adults faring better, being female, which slightly improves survival, as well as the location of the tumour and the extent of tumour resection. More recent studies have shown that further characterisation of tumours by molecular and genetic markers can provide useful prognostic indicators although there is little information in the literature as markers are not routinely recorded for the majority of patients. However, with improved and systematic recording of the molecular characteristics of tumours, the relation to progression and prognosis will be clarified.

\section{CHILDHOOD BRAIN TUMOURS}

Brain tumours are the second most common cancer in children, comprising $15-25 \%$ of all paediatric malignancies, and they are the most common solid tumour. Different

\begin{tabular}{|c|c|c|}
\hline Factor & Specific aspects & Evaluation of risk \\
\hline lonising radiation & Therapeutic, diagnostic & $\begin{array}{l}\text { Therapeutic doses increase risk but diagnostic } x \\
\text { rays do not appear to be associated }\end{array}$ \\
\hline Mobile phones & Radiofrequency exposure & $\begin{array}{l}\text { Current epidemiological and biological evidence } \\
\text { does not support any link between mobile use } \\
\text { and the risk of brain tumours }\end{array}$ \\
\hline $\begin{array}{l}\text { Extremely low frequency } \\
\text { electromagnetic fields }\end{array}$ & $\begin{array}{l}\text { Residential and occupational } \\
\text { exposure }\end{array}$ & Little consistent evidence but research is ongoing \\
\hline$>$ Specific infections & $\begin{array}{l}\text { Viruses, Toxoplasma gondii, } \\
\text { in utero influenza and } \\
\text { varicella }\end{array}$ & $\begin{array}{l}\text { No candidate viruses consistently associated } \\
\text { or found in tumour tissue. Few links to in utero } \\
\text { exposure }\end{array}$ \\
\hline Allergies & Atopy & $\begin{array}{l}\text { The presence of atopy appears to be protective } \\
\text { but further work needed to identify mechanisms }\end{array}$ \\
\hline$>$ Diet & $\begin{array}{l}\text { Nitrosamine/nitrosamide/ } \\
\text { nitrite/nitrate consumption. } \\
\text { Aspartame }\end{array}$ & No consistent evidence \\
\hline$>$ Tobacco & Cigarettes, cigars, pipes & No associations \\
\hline Alcohol & & No associations \\
\hline Chemical agents & $\begin{array}{l}\text { Hair dyes, solvents, pesticides, } \\
\text { traffic related air pollution }\end{array}$ & No consistent evidence \\
\hline Occupations & $\begin{array}{l}\text { Rubber manufacture, vinyl } \\
\text { chloride, petroleum refining }\end{array}$ & $\begin{array}{l}\text { Small risks associated with working in the } \\
\text { petroleum/oil industry but no mechanism } \\
\text { or specific chemical known }\end{array}$ \\
\hline Head trauma/injury & & No consistent evidence \\
\hline
\end{tabular}


proportions of histological subtypes are present in children compared to adults, with gliomas (approximately 40\%) and medulloblastomas (approximately 25\%) mainly arising infratentorially, with the remainder, germ cell tumours and craniopharyngiomas, occurring in the midline. There is a small peak in incidence in early childhood accounted for by medulloblastomas. Studies in the USA, Sweden, and the UK have reported what appear to be true rises in incidence over the last three decades which are unexplained by changes in diagnostic practice, treatment, or classification. The question of whether this is a real and continuing effect can only be answered by future surveillance using accurate and complete population based registers.

Survival for children with brain tumours has improved by $16 \%$ since 1971 in England and Wales. The prognosis in children is superior to that of adults with an overall probability of surviving to five years of 59\% in England and Wales for those diagnosed 1986-90 compared to $72 \%$ in the USA.

\section{RISK FACTORS}

Current thinking suggests that brain tumours develop as a consequence of accumulated genetic alterations that permit cells to evade normal regulatory mechanisms and destruction by the immune system. These alterations may be in part or wholly inherited but any agents-chemical, physical or biological-that damage DNA are possible neurocarcinogens. Investigations of the causes of brain tumours should ideally address the simultaneous influence of both genetic factors and environmental exposures. Table 2 lists the environmental factors which have received attention as possible causes of brain tumours.

\section{GENETICS}

Genetic predisposition to developing brain tumours is associated with certain inherited syndromes such as tuberous sclerosis, neurofibromatosis types 1 and 2, nevoid basal cell carcinoma syndrome, and syndromes involving adenomatous polyps. These syndromes account for $1-2 \%$ of all tumours. The Li-Fraumeni cancer family syndrome is also associated with a predisposition to brain tumours and specifically with mutations in the TP53 gene. Mutations in constitutional (that is, non-tumour tissue) TP53 have been linked to patients with gliomas. Future work on causality should be able to account for possible interactions between known genetic predisposition in the investigation of environmental risk factors.

Familial aggregations of brain tumours occurring in different generations and sibships occur very rarely and the patterns of inheritance are inconsistent. In these situations common environmental exposures cannot be excluded as an explanation. Overall, it appears that only a very small proportion of brain tumours can be attributed to the effect of inherited predisposition.

Common variations in the structure of specific genes are known to be associated with basic cellular metabolic processes such as oxidation, detoxification, DNA stability and repair, and immune functioning. Such genetic polymorphisms may well be associated with the development of brain tumours in the presence or absence of environmental carcinogens. However, the limited findings available so far have failed to consistently identify any specific polymorphisms, but this remains a potentially fruitful line of research and future large sample studies are needed.

\section{IONISING RADIATION}

Ionising radiation given in therapeutic doses is one of the few known risk factors for brain tumours. The now discontinued low dose radiation treatment of tinea capitis and skin disorders in children increased the risk of brain tumours well into adulthood as does radiotherapy for childhood cancers and leukaemia. Survivors of the atomic bomb in Hiroshima have increased risks of meningioma in proportion to their level of exposure. In utero exposure does not appear to affect the risk for the developing fetus. Diagnostic $x$ rays do not appear to be linked to gliomas, but full mouth dental $x$ rays have been associated with meningiomas in a small number of studies.

\section{MOBILE PHONES}

Radiofrequency (RF) signals which fall within the microwave region of the electromagnetic spectrum are emitted and received by mobile phone handsets. The energy levels of these waves are insufficient to damage or disrupt cellular DNA. However, public concern over the possible detrimental health effects of using mobile phones have resulted in a number of investigations of possible links with brain tumours.

When assessing the literature on this topic interpretation of a small number of early studies from the USA and Sweden must be cautious. They were conducted on relatively small populations, relating to a time when analogue phones predominated, they had relatively short follow up periods, or they suffered from methodological shortcomings. To date five further reports from substantial well conducted studies in the USA, Finland, and Denmark using different epidemiological methods have observed the same outcome. They found no increased risk of brain cancers or any subtype of brain tumour associated with exposure to mobile phones using measures of the type of phone, duration and frequency of use, and cumulative hours of use. Thus, so far the consensus of evidence is that mobile phone use does not increase the risk of developing a brain tumour. However, with the exponential increase in the ownership and duration of use of these hand held devices it will be important to continue investigations with respect to digital phones, allowing for a latent period of several decades in the development of a tumour.

\section{EXTREMELY LOW FREQUENCY MAGNETIC FIELDS}

Power frequency extremely low frequency magnetic fields (ELF-MF) of $50-60 \mathrm{~Hz}$ are used in domestic and industrial electricity supplies presenting a virtually ubiquitous exposure to the population, although levels of exposure do vary. The possibility of adverse health effects has generated considerable public concern, despite scant epidemiological and biological support in the research literature. The biological basis for ELF-MF being involved in malignant transformation is not strong as there is no consistent research which shows this low energy exposure has any direct effects on disrupting cellular DNA or metabolic pathways.

Studies of occupational exposure to high levels of ELF-MF and the risk of brain tumours have mixed findings. Variation in results can be explained by differing study methodologies and sample size, the latter affecting the power to detect increased risk. One substantial difference is the method used to assess ELF-MF exposure; this varies from the use of proxies such as wiring configuration codes, distance from distribution equipment, and historic load data to direct measures of exposure. The lack of consistency between 
independent research projects and the difficulties with measuring the exact exposure levels of the individuals at work makes interpretation problematic.

For mothers exposed at work during their pregnancy there does not appear to be any increased risk of their child subsequently developing a brain tumour.

Overhead power cables and wiring configurations in houses affect the levels of exposure to ELF-MF in a domestic residence. Early reports in the late 1980s of childhood brain tumours associated with high levels of domestic exposure have not been replicated. Current evidence shows that at levels experienced by the general population no risk of brain tumours in children appears to be present. However, for extremely high levels of exposure further investigations are underway.

\section{IMMUNE FACTORS: VIRUSES, ALLERGIES, INFECTIONS}

In experimental animal models brain tumours can be induced by a number of viruses, including retroviruses, papovaviruses, and adenoviruses but there is little epidemiological support for this occurring in humans. At one time it was thought that live polio vaccines contaminated with SV40 might increase the risk of brain tumours, but the initial observations were not supported by more detailed powerful studies. Direct examination of brain tumour tissue for evidence of a viral cause has shown the presence of different viral DNA sequences in a proportion of cases within separate pathological series. However, the mechanisms of how a virus might initiate malignant transformation remain unknown and more work is needed to disentangle the putative role of viruses in causing brain tumours.

Atopic diseases such as asthma, eczema, and allergies can be markers of immune dysfunction. In a number of independent studies from different countries atopic conditions have been shown to be "protective", particularly in the development of gliomas. Patients with gliomas report fewer symptoms of atopy compared to control subjects. This relation is an interesting one and might indicate a role for immunologic factors in causation.

In utero infections with influenza and chicken pox (varicella) have been cited as a risk factor but the case for this is not strong. Some recent epidemiological work on a series of children from the north west of England diagnosed with brain tumours has shown geographical distributions which are suggestive of an infectious aetiology for some of the tumour types. Clustering in time and space and seasonality of diagnosis indicate infections may be risk factors.

The involvement of infections and immune responses in brain tumour aetiology is an area of research that clearly warrants further attention.

\section{CHEMICALS}

$\mathrm{N}$-nitroso compounds are found in the environment but the most common source of human exposure is through foods, with vegetables and cured meats being major sources. Certain alkylating agents, such as ethyl and methyl nitrosurea, are known transplacental carcinogens, particularly for brain tumours in rats. Their ability to cross the blood-brain barrier and their mutagenic potential makes them ideal candidates as initiators in the carcinogenic process. In humans, dietary and environmental $\mathrm{N}$-nitroso compounds have been studied as potential brain tumour carcinogens along with the potentially protective effect of consuming antioxidants. The sources of antioxidants include fresh fruit and vegetables, supplements, and endogenous metabolic pathways. Attributing the cause of brain tumours to these compounds or other dietary factors such as vitamin supplements has received mixed support in the published literature. Dietary assessment is fraught with problems and it may be that the ingestion of potentially toxic compounds is offset by the ingestion of antioxidants which promote DNA repair. Nitrate levels in drinking water have also been investigated but no consistent associations found.

The low calorie sweetener aspartame has been commonly used in a number of food products for over 15 years. It has been suggested to be involved in the aetiology of some brain tumours based principally on the results of laboratory experiments. The biological basis for any influence which aspartame could have on the risk of developing a brain tumour is unclear.

Tobacco smoke is carcinogenic but many constituents do not pass the blood-brain barrier. Smoking does not appear to be strongly linked to brain tumours either in adults who smoke themselves or via maternal smoking in pregnancy. A similar lack of association is seen for alcohol consumption.

Various other chemicals have received attention. Hair dyes and hair sprays were implicated as risk factors for brain tumours in some early epidemiological studies but the observations remain unconfirmed. Further inconsistent reports have linked childhood brain tumours to pesticide exposure, traffic pollution, and parental occupations. The possibility of fathers' sperm being damaged and the developing fetus being affected by parental occupation and the development of childhood brain tumours has been extensively studied, but few conclusions have been drawn. A recent large scale case-control study of childhood cancers in the UK failed to show any significant associations between brain tumours and the occupations of either mothers during pregnancy or fathers around the time of conception.

In the working population many jobs in various industries involve exposure to carcinogenic or neurotoxic compounds including organic solvents, polycyclic aromatic hydrocarbons, lubricating oils, and phenols and the question has been frequently asked as to whether such exposure is related to brain tumours. Despite numerous studies no consistent risks have been isolated for any chemical or group of workers apart from those in the petrochemical and oil industry. In these circumstances no specific chemical has been identified and the possibility of multiple exposures has to be considered.

\section{HEAD TRAUMA AND INJURY}

Patients with brain tumours inevitably recall occurrences of trauma or injury to the head with greater frequency than the general population, and studies of patients' reports are therefore subject to "recall bias". Some epidemiological investigations of the relation between head trauma/injury and the subsequent development of a tumour have attempted to overcome this by examining medical records, but these mainly fail to demonstrate any relation. The inevitable pitfall of recall bias as an explanation of a raised risk renders most work in this area virtually impossible to interpret.

\section{SUMMARY}

Brain tumours in adults are a rare disease from which survival is generally poor compared to many other cancers. Reports of rising trends need to be cautiously interpreted as 
they may well be explained by changes in diagnostic and clinical practice. In childhood a different profile of tumour types is present and survival has improved over recent years and is higher than in adults. Apart from genetic predisposition, the most well established environmental risk factor for brain tumours is exposure to high doses of ionising radiation. Research into infections and immune factors may prove a fruitful avenue of investigation.

\section{SOURCES OF INFORMATION}

International

$\checkmark$ The International Agency for Research on Cancer (www.iarc.fr)

- Central Brain Tumour Registry of the United States (CBTRUS) (www.cbtrus.org)

\section{The UK}

- Cancer Research UK (www.cancerresearchUK.org )

- UK Association of Cancer Registries (UKACR) (www. UKACR.org.uk)
- England: National Cancer Intelligence Centre, Office for National Statistics (www.statistics.gov.uk)

- Wales: Welsh Cancer Intelligence and Surveillance Unit (www.velindre-tr.wales.nhs.uk)

- Scotland: Information and Statistics Division, NHS in Scotland (www.show.scot.nhs.uk/isd)

- Northern Ireland: Northern Ireland Cancer Registry (www.qub.ac.uk/nicr/intro.htm)

\section{REFERENCES}

1 Coleman MP, Babb P, Damiecki D, et al. Cancer survival trends in England and Wales, 1971-1995: deprivation and NHS region. Studies in medical and population subjects no. 61. London: Stationery Office, 1999.

2 Davis FG, McCarthy BJ. Current epidemiological trends and surveillance issues in brain tumours. Expert Rev Anticancer Ther 2001;1:395-401.

3 Little J. Epidemiology of childhood cancer. IARC Scientific Publication no. 149. Lyon, France: International Agency for Research on Cancer, 1999.

4 McKinney PA, Fear NT, Stockton D, on behalf of the UK Childhood Cancer Study Investigators. Parental occupation at periconception: findings from the United Kingdom childhood cancer study. Occup Environ Med 2003;60:901-9.

5 Wrensch $M$, Minn $Y, C$ Chew T, et al. Epidemiology of primary brain tumours: current concepts and review of the literature. Neuro-oncology 2002:4:278-99. 